http://jmscr.igmpublication.org/home/ ISSN (e)-2347-176x ISSN (p) 2455-0450 crossref DOI: https://dx.doi.org/10.18535/jmscr/v7i8.48

Journal Of Medical Science And Clinical Research

\title{
Rare case report of Rhinosinusitis by Aspergillus flavus and Rhizopus in a diabetic patient
}

\author{
Authors \\ Dr Disha Sharma ${ }^{1}$, Dr Vineeta Sharma*2, Dr Santwana Verma ${ }^{3}$, Dr Anumeha Gupta ${ }^{4}$, \\ Dr Parul Sharma ${ }^{5}$ \\ ${ }^{1}$ Medical Officer Specialist (Otorhinolaryngology) Dr Radhakrishnan Govt. Medical College, Hamirpur \\ ${ }^{2}$ Assistant Professor, Microbiology, Indira Gandhi Medical College, Shimla \\ ${ }^{3}$ Professor Microbiology, Indira Gandhi Medical College Shimla \\ ${ }^{4,5}$ Junior Resident, Microbiology, Indira Gandhi Medical College Shimla \\ *Corresponding Author \\ Dr Vineeta Sharma
}

Assistant Professor, Microbiology Indira Gandhi Medical College Shimla, Himachal Pradesh, India

\begin{abstract}
The fungal rhinosinusitis is being reported increasingly these days. The common causative agents are Aspergillus and Zygomycetes. It can affect immunocompetent as well as immunocompromised individuals. Dual infection of paranasal sinuses with both Aspergillus and Rhizopus is a rare presentation. We are reporting such a rare case of elderly male with uncontrolled type 2 diabetes mellitus who presented with fungal rhinosinusitis having coinfection by Aspergillus and Rhizopus.

Keywords: Rhinosinusitis, Aspergillus, Rhizopus, Diabetes mellitus.
\end{abstract}

\section{Introduction}

Fungal rhinosinusitis was thought to be a rare entity in the past. This condition is being reported increasingly nowadays ${ }^{(1)}$. This could be due to improvement in diagnostic techniques, increased awareness about the fungal infections and an increase in immunocompromised conditions like increased use of broad spectrum antibiotics and corticosteroids, chronic diseases as diabetes, malignancies. Though fungal infections of paranasal sinuses can affect both immunocompetent and immunocompromised individuals. Immunosuppresion is associated with more serious and invasive disease. Various fungal agents isolated from sinuses are Aspergillus, Mucor, Penicillium Candida, Acremonium, Fusarium, Curvularia etc. Among all these
Aspergillus and Zygomycetes are the commonest. These fungi have angioinvasive property so they can invade and occlude blood vessels leading to infarction of important organs. So these patients require early diagnosis and treatment. Reports of coinfection by Aspergillus and Rhizopus are very rare. We are here describing such a case with dual infection by Aspergillus and Rhizopus both, in a diabetic patient.

\section{Case Report}

A 62 years old male presented to our department with complaint of headache and swelling on left side face since 7 days. He also had decreased left eye movement with diminished vision of left eye for the last 5 days. He also complained of left sided nasal obstruction since last 3 days. At the 
time of presentation patient was well oriented. He was a known case of Type 2 diabetes mellitus for the past 5 years. He was on insulin treatment but at the time of presentation his diabetes was not controlled. His blood sugar was $447 \mathrm{mg} / \mathrm{dl}$ and glycosylated haemoglobin was 13.8 . There was no history of fever and vomiting.

On examination there were blackish crusts in the left nasal cavity with right sided deviation of nasal septum. Eye examination revealed complete ophthalmoplegia of the left eye with no perception of light and the pupil was not reacting to light. Other blood investigations revealed $\mathrm{Hb}$ (Haemoglobin) 13g/dl, WBC (white blood cell) count of $13,500 / \mathrm{cmm}$ and ESR was $60 \mathrm{~mm} / 1 \mathrm{st}$ hour. MRI, T2 weighted, FLAIR and contrast enhanced T1weighted images showed left maxillary sinusitis with its partial opacification. Further extension to involve left nasal cavity, bilateral ethmoid sinuses and sphenoid sinus. (Figure 1a and $1 \mathrm{~b}$ ).

Patient was taken for endoscopic sinus surgery. Intraoperatively there was blackish crust on nasal mucosa, septum, floor and lateral nasal wall. There was necrosed tissue and blackish eschar seen to be extending to maxillary and (Figure 2) sphenoid sinus. Debrided tissue was collected and sent for histopathological and microbiological diagnosis.

Grossly tissue revealed black and necrosed mass. It was minced for $\mathrm{KOH}$ mount preparation which revealed two types of fungal elements. (Figure 3). There were wide ribbon like aseptate hyphae with sporangia. Surprisingly there were collapsed umbrella shaped sporangia also. There were also seen branched narrow septate hyaline hyphae. The tissue was minced and inoculated onto SDA (Sabouraud dextrose agar)with antibiotics and incubated at $25^{\circ} \mathrm{C}$ and $37^{\circ} \mathrm{C}$. After 2 days two types of growth was noticed. One of the growth was woolly, fluffy and the other was granular with yellowish green colour. LCB mount preparation also showed two types of fungal elements(Figure 4). These findings confirmed the dual infection by Aspergillus flavus and Rhizopus. Patient was started on intravenous Amphotericin B and showed improvement on treatment.
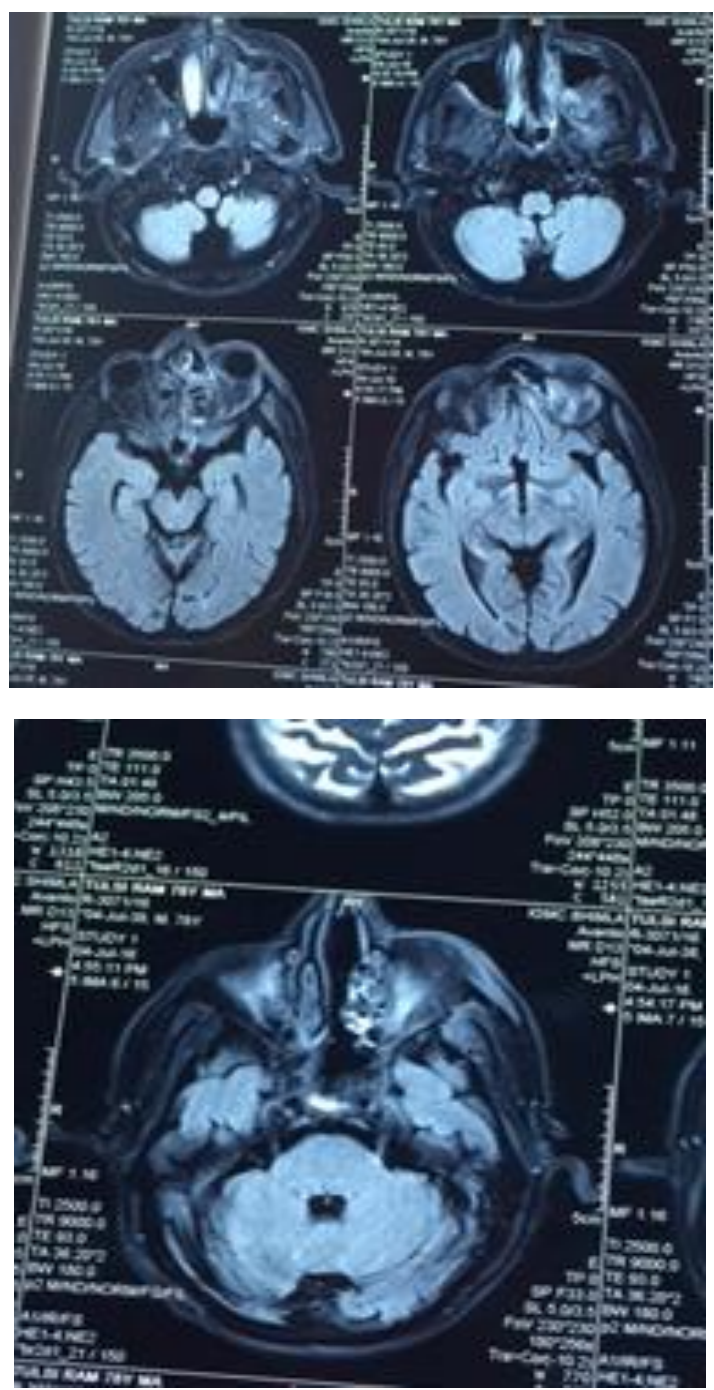

Figure 1a and 1b: MRI showing partial opacification of left maxillary sinus with involvement of bilateral ethmoid sinuses and sphenoid sinus.

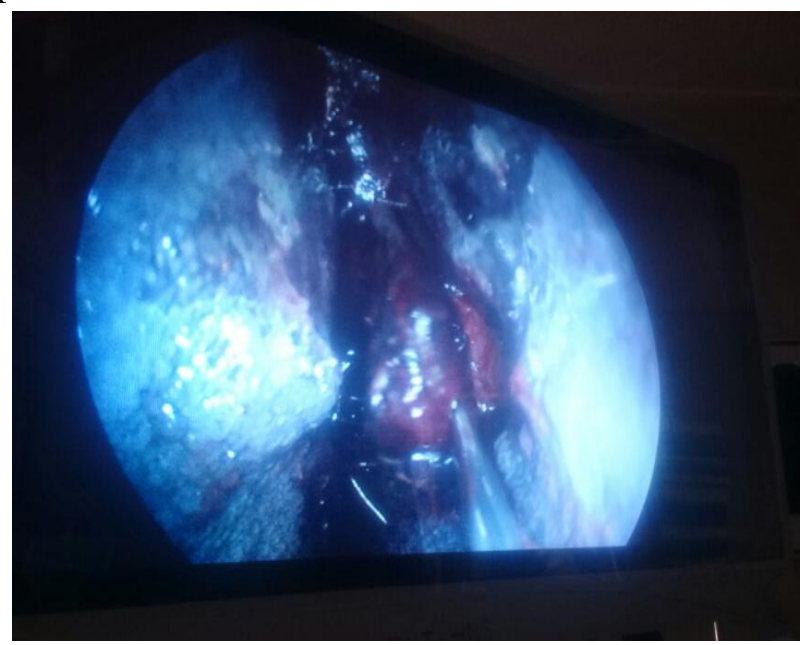

Fig 2 Intraoperative Endoscopic image showing necrosed tissue with blackish eschar. 


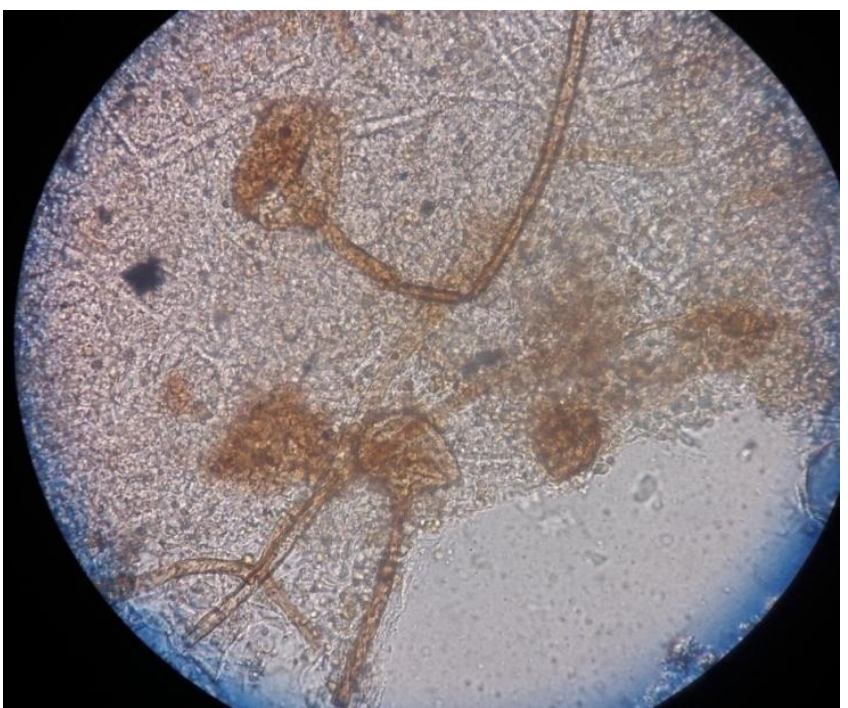

Figure 3: $\mathrm{KOH}$ mount showing broad aseptate hyphae with collapsed sporangia and thin hyaline septate hyphae with branching.

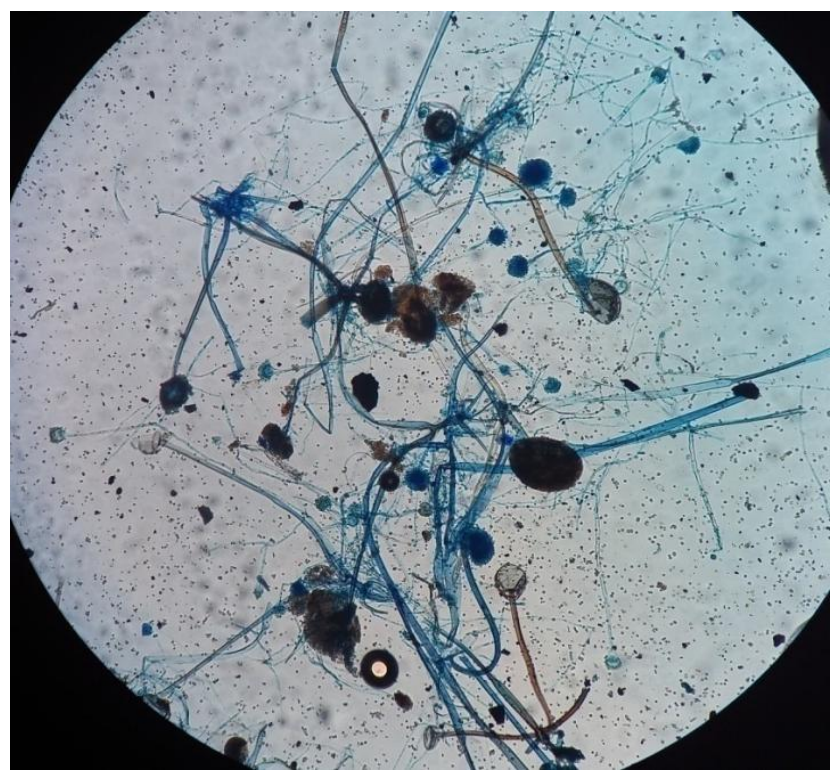

Figure 4: LCB mount from mixed culture showing two types of fungi identified as Rhizopus and Aspergillus flavus.

\section{Discussion}

Fungal sinusitis was first reported in 1791AD by Plaignaud in a 22 year old male ${ }^{(2)}$. The frequency of mycotic infections of paranasal sinuses is increasing these days globally ${ }^{(1)}$. Different fungi associated with fungal sinusitis are Aspergillus species, Curvularia, Bipolaris, Exserohilum and zygomycetes. of all these Aspergillus and zygomycetes are the commonest. We are reporting a rare case of coinfection by both Aspergillus and Rhizopus involving paranasal sinuses. On extensive literature review we could find only few such publications by Mairano et al, Rit et al, Alfano et al and Vaidya D..$^{(3,4,5,6)}$

Aspergillus is a fungus of ascomycetes class whereas Rhizopus belongs to phycomycetes class. Both are saprophytic fungi commonly found in decaying vegetable and soil. They release airborne spores which easily find their ways to sinusoidal cavities through respiration. They are commonly found colonising the oral mucosa,nose and paranasal sinuses.

Globally the most frequent species isolated from case of sinusitis is Aspergillus fumigatus followed by Aspergillus flavus. The sinus involved frequently is maxillary sinus.

The fungi causing mucormycosisnhave angioinvasive property so they can form thrombi and result in ischemia and infarction of affected organ. The infection can spread to adjacent sinuses and into orbit. Patient can even have unilateral to bilateral visual loss due to cavernous sinus thrombosis. ${ }^{(7)}$

Various factors responsible for promoting fungal infections of paranasal sinuses include polyps, stagnant secretions and underlying immunecompromising conditions like corticosteroids, uncontrolled diabetes, HIV infection, malignancies, burns and radiation therapy ${ }^{(8)}$. The patient in present case also had uncontrolled diabetes which must have contributed for his presentation. The normal immunological response to infections is altered in uncontrolled diabetes.

They have decreased granulocytic phagocytic activity. The polymorphonuclear response in such patients is also altered. It has been found that immunocompromised patients are suitable hosts for fungal infections as ability of serum of such patients to inhibit Rhizopus in vitro has been found to be reduced. Moreover the acidosis and increased blood sugar levels provide an excellent environment for the fungus to grow ${ }^{(9)}$.

The radiological investigations, MRI (Magnetic resonance imaging) and CT (Computerised tomography) scan, help to establish the diagnosis of invasive fungal sinusitis by demonstrating 
opacity of sinus with or without destruction. MRI is more sensitive for intraorbital and intracranial extension, whereas CT scan gives more detail about bony changes ${ }^{(10)}$. These clinical and radiological findings require the final biopsy report to establish the diagnosis. Microscopic findings of hyaline hyphae with branching at $45^{\circ}$ angles is seen in Aspergillus and broad nonseptate ribbon like hyphae are suggestive of Zygomyecetes. Final confirmation is only after the fungal culture is obtained on SDA.

The management of such patients require early diagnosis and aggressive treatment because the patient has already suffered significant tissue damage by the time of presumptive diagnosis. Patients require both surgical and medical therapies. Aggressive debridement of the affected area and appropriate antifungal therapy plays an important role. The usual drug of choice is lipid formulation of Amphotericin B at the dose of $5 \mathrm{mg} / \mathrm{kg}$ IV per day. The duration depends on location of infection, underlying disease and response to therapy. Patient with underlying predisposing factors as diabetes require that the blood sugar levels are optimally controlled. Our patient was also managed by first controlling his blood sugar levels followed by surgical debridement along with Amphotericin B. According to 2008 IDSA (Infectious disease society of America) guidelines Voriconazole has now become the drug of choice for invasive fungal sinusitis due to better tolerance, increased efficacy and less toxicity. ${ }^{(11)}$

We reported the case to highlight the presence of coinfection of paranasal sinuses by Aspergillus and Rhizopus and the importance of early diagnosis and management of fungal rhinosinusitis by combined efforts of clinician ,radiologist and microbiologist. The successful treatment of invasive aspergillosis with mucormycosis is early diagnosis, followed by aggressive surgical debridement along with appropriate antifungal agent.

\section{References}

1. Chakrabarti A, A.Das, JMandal et al.,"The rising trend of invasive zygomycosis in patients with uncontrolled diabetes mellitus," Medical Mycology 2006 ;vol.44,no. 4: 335-342

2. Rodrigues J, Caruthers C, Azmeh R , Dykewicz M.S, Slavin R.G, Knutsen A.P. The spectrum of allergic fungal diseases of the upper and lower airways. Expert Rev. Clin. Immunol 2016;12:531-550.

3. Maiorano E, Favia G, Capodiferro $S$, Montagna M T, Lo Muzio L. Combined mucormycosis and aspergillosis of orosinonasal region in a patient affected by Castleman disease. Virchows Arch 2005;446:28-33

4. Rit K, Saha R, Dey R, B Gautam. Rhinooculo-cerabral aspergillus and mucor coinfections in an immunocompromised patient with type 2 diabetes mellitus. Medical Journal of Dr. D.Y.Patil University 2014;7:4486-88.

5. Alfano C, Chiummariello S, Dessy LA, BistoniG, Scuuuuuuuuuderi N. Combined Mucormycosis and Aspergillosis of the Rhinocerebral Region. In vivo 2006;20:311-16

6. Vaidya D, Shah P. Coinfection by Aspergilus and Zygomycetes Species in a case of Acute Rhinosinusitis. Case reports in Otolaryngology 2011;Article ID 382473,5 pages.

7. Gupta R, Gupta B, Bal A, Gupta AK. Sinonasal mucormycosis with Fungal ball: A Rare Case Report. Clinical Rhinology: An International Journal, MayAugust 2014;7(2):64-66.

8. Singh N, Siddaraju N, Kumar S, Muniraj F, Bakshi S, Gopalakrishnana S. Fine needle aspiration biopsy as an initial diagnostic modality in a clinically unsuspected case of invasive maxillary fungal sinusitis: Acase report. Diagn Cytopathol. 2010;38:290-3. 
9. Khan AR, Khan MU, Ullah S, Ullah H, ShahJ. Rhinocerebral mucormycosis. J Coll Physicians Surg Pak 2002;12:639-41.

10. Sharma D, Mahajan N, Rao S, Khurana N, Jain S. Invasive maxillary aspergillosis masquerading as malignancy in two cases utility of cytology as a rapid diagnostic tool. J Cytol. 2012;29:194-6.

11. Arakawa H, Suto C, Notani H, Ishida T, Abe K, Ookubo Y. Selection of antifungal agent decides prognosis of invasive aspergillosis: case report of a successful outcome with voriconazole. Int Ophthalmol 2014;34:85-9. 